\title{
Bilateral Comparisons and Consistent Fair Division Rules in the Context of Bankruptcy Problems ${ }^{1}$
}

\author{
NiR DAGAN \\ Department of Economics, The Hebrew University of Jerusalem, Mount Scopus, 91905 Jerusalem, \\ Israel
}

OSCAR VOLIJ ${ }^{2}$

Department of Economics, Box B, Brown University, Providence, Rhode Island 02912, USA

Abstract: We analyze the problem of extending a given bilateral principle of justice to a consistent $n$-creditor bankruptcy rule. Based on the bilateral principle, we build a family of binary relations on the set of creditors in order to make bilateral comparisons between them. We find that the possibility of extending a specific bilateral principle of justice in a consistent way is closely related to the quasi-transitivity of the binary relations mentioned above.

\section{Introduction}

The principle of bilateral comparisons is central in the theory of social and individual choice. Clearly, in order to make bilateral comparisons a binary relation is needed. However, if we want these bilateral comparisons to yield a sensible choice rule, we need to impose restrictions on the binary relation on which the pairwise comparisons are based. Sen (1969) showed, for instance, that a sufficient condition for a complete binary relation to generate a choice function is that this binary relation be quasi-transitive. ${ }^{3}$ To interpret this result, note that any complete binary relation induces a choice function for 2-alternative choice problems. Sen's result tells us that this 2-alternative choice function can be extended to any finite-alternative choice function if the binary relation we started with is quasi-transitive.

An alternative use of the principle of bilateral comparisons has been suggested in the context of fair division problems (see, for example, Harsanyi (1959) and Lensberg (1987)): An outcome in an $n$-person division problem is not considered fair if it is unfair to any particular pair of individuals. This alternative use of the

\footnotetext{
${ }^{1}$ We would like to thank Bhaskar Dutta, Vincent Feltkamp, Yoram Halevy, Sergiu Hart, Sjaak Hurkens, Ko-Ichi Tadenuma, Eyal Winter and an anonymous referee for their helpful comments.

2 The main part of this paper was written when this author was at CentER for Economic Research, Tilburg.

${ }^{3}$ Quasi-transitivity of a complete binary relation is equivalent to the transitivity of its asymmetric part.
} 
principle of bilateral comparisons assumes that we can define a fair outcome when the fair division problem involves only two individuals. That is, that we have a principle that singles out a fair division in two-person problems. The question is: are there any conditions that allow us to extend this two-person principle to a finite-person fair decision rule, in a consistent way.

Our main result is that the alternative use of the principle of bilateral comparisons is also related to quasi-transitivity when applied to allocation rules in the context of bankruptcy problems. We found that in order to be able to extend a 2-person bankruptcy rule to an $n$-person bankruptcy rule in a consistent manner, the 2-person rule should define a quasi-transitive binary relation on the set of creditors, for any feasible allocation.

In order to prove our result, we define a family of bankruptcy rules, which is interesting in itself. This is the family of bankruptcy rules that are "consistent on average". As consistency requires that the outcome should be fair when comparing any two individuals, "consistency on average" requires that the outcome should be fair only on average, after having carried out all the pairwise comparisons. We show that any given two-person bankruptcy rule has a unique extension which is consistent on average. Furthermore, if the initial 2-person rule happens to have a consistent extension, then the consistent-on-average rule is precisely this consistent extension. Moreover, given the bilateral rule, the recommendation given by the average consistent extension to any specific bankruptcy problem can easily be computed based only on the bilateral principle of comparisons that generated it. The importance of this family of rules is that they are a natural generalization of the consistent rules, but unlike them, there is always an average consistent rule with respect to any arbitrary bilateral principle of comparison.

Our paper is related to Young (1987) who shows that a symmetric and continuous bankruptcy rule is consistent if and only if it is representable by a continuous parametric function. From this we can conclude that a symmetric and continuous 2-person rule can be extended in a consistent way if and only if it has a parametric representation. Moreover, a parametric representation of a rule induces an interval representation à la Fishburn (1970) of our binary relation on the set of creditors. Young (1987) also shows that a symmetric and continuous bankruptcy rule is consistent if and only if its recommendations maximize a symmetric, continuous, additively separable and strictly concave function. All these results give alternative insights on the concept of consistency, which proved central in the analysis and characterization of many prominent solution concepts. We believe that the main result of our paper gives another interesting view on the concept of consistency.

The paper is organized as follows. Section 2 gives the formal treatment of bankruptcy problems. Section 3 deals with bilateral principles of justice and shows how they can be used to evaluate the fairness of allocations. The main result on the relation between consistency and the quasi-transitivity of the binary relations generated by the bilateral principles is also stated in this section. Section 4 presents the concept of consistency on average and shows that together 
with any monotone bilateral principle it characterizes a unique bankruptcy rule, which is further used to prove the main result. Other results concerning strictly monotone rules are also shown. Section 5 discusses the role of the assumptions and the relation between two different extensions of a bilateral principle which has no consistent generalization. Section 6 concludes.

\section{The Bankruptcy Model}

A bankruptcy problem is a pair $(E ; \mathbf{d})$ where $\mathbf{d} \in \mathbb{R}_{+}^{I}$ is a vector of nonnegative real numbers (the claims), which are indexed by some finite nonempty subset $I$ of natural numbers (the creditors), and $0 \leq E \leq \sum_{i \in I} d_{i}=: D . E$ is the estate to be allocated, and $D$ is the sum of the claims.

An allocation in $(E ; \mathbf{d})$ is a vector $\mathbf{x} \in \mathbb{R}_{+}^{I}$ such that $\sum_{i \in I} x_{i}=E$ and $x_{i} \leq d_{i}$ for all $i \in I$. The set of all allocations in $(E ; \mathbf{d})$ will be denoted by $A(E ; \mathbf{d})$.

Remark: For any list of claims $\mathbf{d} \in \mathbb{R}_{+}^{I}$, any vector $\mathbf{x} \in \mathbb{R}_{+}^{I}$ with $x_{i} \leq d_{i}$ is an allocation of the bankruptcy problem $\left(\sum_{i \in I} x_{i} ; \mathbf{d}\right)$. Therefore, when there is no danger of confusion, we shall call any such vector $\mathbf{x}$ an allocation without specifying the bankruptcy problem to which it refers.

A rule is a function that assigns to each bankruptcy problem a unique allocation.

Examples:

a) The proportional rule is defined as follows:

$\operatorname{Pr}(E ; \mathbf{d})=\lambda \mathbf{d}, \quad$ where $\lambda D=E$.

The proportional rule allocates awards proportionally to the claims.

b) The constrained equal award (CEA) rule is defined as follows:

$\operatorname{CEA}(E ; \mathbf{d})=\mathbf{x}$, where $x_{i}=\min \left(\lambda, d_{i}\right)$ and $\lambda$ solves the equation $\sum_{i \in I} \min \left(\lambda, d_{i}\right)=E .^{4}$

This rule assigns the same sum to all creditors as long as it does not exceed each creditor's claim.

${ }^{4}$ This equation has a unique solution when $D>E$. If $D=E$, any solution $\lambda$ is greater than or equal to the maximum claim and therefore $x_{i}=d_{i}$ for all $i$. 
The excess of a claim over the estate is completely ignored by the CEA rule. The proportional rule, on the other hand, takes into account the entire claim. The following example shows a rule that combines the proportionality principle with the principle that excesses of claims over the estate should be ignored.

c) The modified proportional (MP) rule is defined as follows:

$$
\operatorname{MP}(E ; \mathbf{d})=\operatorname{Pr}(E ;(\mathbf{d} \wedge \mathbf{E})),
$$

where the $i$ th component of the vector $\mathbf{d} \wedge \mathbf{E}$ is $\min \left\{d_{i}, E\right\}$.

The MP rule allocates awards proportionally to the relevant claims. For a cooperative bargaining motivation of this rule see Dagan and Volij (1993, Proposition 3).

A bilateral principle is a function that assigns to each 2-creditor bankruptcy problem a unique allocation.

Formally, the restriction of any rule to the class of 2-creditor bankruptcy problems is a bilateral principle (we shall call it the bilateral principle associated to the rule). For reasons that will become clear in the next section, however, we want to make a distinction between a 2-creditor rule and the corresponding bilateral principle. For this reason, we are going to use the greek letter $\phi$ to denote an allocation rule and the latin letter $f$ to denote its associated bilateral principle.

Next, we present three properties of allocation rules.

A rule $\phi$ is monotone if for all $(E ; \mathbf{d})$ and $0 \leq E^{\prime} \leq E, \phi\left(E^{\prime} ; \mathbf{d}\right) \leq \phi(E ; \mathbf{d})$. Monotonicity says that a decrease in the estate does not benefit any creditor. A rule $\phi$ is strictly monotone if for all $(E ; \mathbf{d})$ and $0 \leq E^{\prime}<E$, if $d_{i}>0$ then $\phi_{i}\left(E^{\prime} ; \mathbf{d}\right)<\phi_{i}(E ; \mathbf{d})$. Strict monotonicity says that a decrease in the estate makes every non-zero creditor worse off.

A rule $\phi$ is anonymous if for all bankruptcy problems $(E ; \mathbf{d})$ and for all permutations $\sigma$ of the set of players $I, \phi_{\sigma(i)}(E ; \mathbf{d})=\phi_{i}(E ; \sigma(\mathbf{d}))$ where the vector of claims $\sigma(\mathbf{d})$ is defined by $\sigma_{i}(\mathbf{d})=d_{\sigma(i)}$. Anonymity requires that the awards should not depend on the names of the players. Monotonicity and anonymity of bilateral principles are defined in a similar and obvious way.

Let $(E ; \mathbf{d})$ be a given bankruptcy problem with set of creditors $I$. For each nonempty subset of creditors $J$ and for each allocation $\mathbf{x}$ in $(E ; \mathbf{d})$ the reduced bankruptcy problem of $J$ with respect to $\mathbf{x}$ is $\left(\sum_{j \in J} x_{j} ; \mathbf{d} \mid J\right)$, where $\mathbf{d} \mid J$ is the restriction of $\mathbf{d}$ to $\mathbb{R}_{+}^{J}$.

A rule $\phi$ is consistent if for any finite nonempty set $I$ of creditors

for all $(E ; \mathbf{d}), \mathbf{d} \in \mathbb{R}_{+}^{I}$, for all $\varnothing \neq J \subset I$,

$$
\phi(E ; \mathbf{d})=\mathbf{x} \Rightarrow \mathbf{x} \mid J=\phi\left(\sum_{i \in J} \mathbf{x}_{i}, \mathbf{d} \mid J\right) .
$$

A rule $\phi$ is consistent if for each bankruptcy problem $(E ; \mathbf{d})$ and subset $J$ of creditors, if $\phi$ chooses $\mathbf{x}$ in $(E ; \mathbf{d})$, then $\phi$ chooses $\mathbf{x} \mid J$ in the reduced bankruptcy 
problem of $J$ with respect to $\mathbf{x}$. The interpretation of consistency is as follows. Suppose that a rule $\phi$ assigns allocation $\mathbf{x}$ to the bankruptcy problem $(E ; \mathbf{d})$. Suppose, too, that some subset of creditors wants to reallocate the total amount $\sum_{i \in J} x_{i}$ assigned to it. If we apply the same rule $\phi$ to allocate this amount among this subset of creditors, each one will get the amount originally assigned to him, provided $\phi$ is consistent. Consistency in the setup of bankruptcy problems was first discussed by Aumann and Maschler (1985) and further analyzed by Young $(1987,1988)$. All the rules presented in the above examples are monotone and anonymous. Strict monotonicity is not satisfied by the CEA rule, and the MP rule is not consistent.

Consistency is a property that relates solutions to bankruptcy problems with distinct number of creditors and therefore consistency cannot be defined for bilateral principles. It turns out, however, that the consistency of a rule $\phi$ is very much related to the existence of very special allocations which are defined solely in terms of the bilateral principle associated to $\phi$. To state this relation we need the following definition:

Let $(E ; \mathbf{d})$ be a bankruptcy problem and let $f$ be a bilateral principle. An allocation $\mathbf{x}$ in $(E ; \mathbf{d})$ is said to be $f$-just if for all creditors $i \neq j$, the division of $x_{i}+x_{j}$ recommended by $f$ for claims $d_{i}$ and $d_{j}$ is precisely $\left(x_{i}, x_{j}\right)$.

Remark 2.1: It follows directly from the definition of consistency that if $\phi$ is consistent, then in every bankruptcy problem there exists an $f$-just allocation, where $f$ is the restriction of $\phi$ to the class of 2-creditor problems. What is not so obvious is that when the bilateral principle is monotone, the converse is also true. Namely, if for every bankruptcy problem there exists an $f$-just allocation, this $f$-just allocation is unique and the bankruptcy rule $\phi$ which assigns each bankruptcy problems its unique $f$-just allocation, is consistent. This result is a simple extension of Aumann and Maschler (1985) Theorem A and Corollary 3.1. For this reason, we are going to restrict attention to monotone bilateral principles.

Before we move on to the main question we need one more definition.

Let $f$ be a bilateral principle, an allocation rule $\phi$ is a consistent extension of $f$ if $\phi$ is consistent and coincides with $f$ for all 2-creditor bankruptcy problems 5 .

\section{On Bilateral Comparisons and Consistency}

The purpose of this paper is to find necessary and sufficient conditions on a bilateral principle $f$ that guarantee the existence of a consistent extension of it. In order to answer this question we are going to use the bilateral principle for bilateral comparisons. We are going to interpret the division recommended by

\footnotetext{
${ }^{5}$ In a different context, Thomson (1994) uses the term consistent extension with a different meaning.
} 
the bilateral principle as the "fair" division in a 2-creditor problem. This fair division will enable us to compare the treatment any two players get at any given allocation.

Given a bilateral principle $f$, a list of claims $\mathbf{d}$ and an allocation $\mathbf{x} \leq \mathbf{d}$, we can define the following binary relation on the set of creditors I:

$$
\succ_{\mathrm{x}}=\left\{(i, j) \in I \times I \mid f_{i}\left(x_{i}+x_{j} ;\left(d_{i}, d_{j}\right)\right)<x_{i}\right\}^{6}
$$

$i \succ_{\mathbf{x}} j$ means that $\mathbf{x}$ treats $i$ better than $j$ according to $f$, or more shortly, $\mathbf{x}$ treats if $f$-better than $j$. Obviously, if $i \succ_{\mathrm{x}} j$ then $f_{j}\left(x_{i}+x_{j} ;\left(d_{i}, d_{j}\right)\right)>x_{j}$. Note that if $i \succ_{\mathrm{x}} j$, then $i \succ_{\mathrm{y}} j$ for any other allocation $\mathrm{y}$ in which $y_{i}=x_{i}$ and $y_{j}=x_{j}$. That is, whether $i$ is treated $f$-better than $j$ or not is independent of the amounts assigned by $\mathbf{x}$ to other creditors. Further note that $\succ_{\mathrm{x}}$ is irreflexive i.e., nobody can be treated better than himself.

We define the relations $z_{x}$ and $\sim_{x}$ by replacing $<$ in the definition of $\rangle_{x}$ with $\leq$ and $=$ respectively. Clearly, $z_{\mathrm{x}}$ is a complete binary relation. Following Sen (1969), we say that $\succ_{x}$ is quasi-transitive if and only if $\succ_{x}$ is transitive. Transitivity of $\succ_{x}$ will play a central role in what follows. The above relations have the obvious interpretation. In particular, we shall say that an allocation $\mathbf{x}$ treats creditors $i$ and $j f$-equally if $i \sim_{\mathrm{x}} j$. We can now restate the definition of an $f$-just allocation by saying that it treats every two creditors $f$-equally.

An appealing feature of $f$-just allocations is that no creditor can complain about being treated worse than any other one. Unfortunately, there are bilateral principles $f$ with no consistent generalization, that is, for some bankruptcy problems $f$-just allocations cannot be found. Consider for example the bankruptcy problem $(E ; \mathbf{d})=((400 ; 100,200,300)$. When the bilateral principle is the MP rule, there is no $f$-just allocation in it. This will follow from our Theorem 3.2 after taking into account that for $\mathbf{x}=(70.42,134.08,195.50)$, the relation $>_{\mathrm{x}}$ is not transitive. However, when $f$-just allocations exist, they can be used as a standard of comparison between players, as the following lemma states:

Lemma 3.1: (Dagan, Serrano and Volij, 1994, Lemma 3.3): Let $f$ be a monotone and anonymous principle and let $(E$; d) be a bankruptcy problem. Assume that there exists an $f$-just allocation in $(E ; \mathbf{d})$ and denote it by $\mathbf{x}^{*}$. Let $\mathbf{x}$ be an allocation in $(E ; \mathbf{d})$ in which there are two creditors $i$ and $j$ with $x_{i} \leq x_{i}^{*}$ and $x_{j} \geq x_{j}^{*}$. Then, $j \succeq_{\mathrm{x}} i$. Moreover, if both inequalities are strict, then $j \succ_{\mathrm{x}} i$.

\section{Proof:}

Case 1: $x_{i}+x_{j} \geq x_{i}^{*}+x_{j}^{*}$. By monotonicity and $f$-justice,

$$
f_{i}\left(x_{i}+x_{j} ;\left(d_{i}, d_{j}\right) \geq f_{i}\left(x_{i}^{*}+x_{j}^{*} ;\left(d_{i}, d_{j}\right)\right)=x_{i}^{*} \geq x_{i} . \text { Hence, } j \geq_{\mathbf{x}} i .\right.
$$

${ }^{6}$ For $i=j$, we define $f_{i}\left(x_{i}+x_{j} ;\left(d_{i}, d_{j}\right)\right)$ to be $x_{i}$. 
Case 2: $x_{i}+x_{j}<x_{i}^{*}+x_{j}^{*}$. By monotonicity and $f$-justice,

$$
f_{j}\left(x_{i}+x_{j} ;\left(d_{i}, d_{j}\right) \leq f_{j}\left(x_{i}^{*}+x_{j}^{*} ;\left(d_{i}, d_{j}\right)\right)=x_{j}^{*} \leq x_{j} . \text { Hence, } j \gtrless_{\mathrm{x}} i .\right.
$$

This proves the first part of the claim. As for the second part, it is proved analogously and is left to the reader.

If we are going to use a bilateral principle $f$ for bilateral comparisons, it is desirable that the relations $\succ_{\mathrm{x}}$ it defines should be transitive. For if we find an allocation $\mathbf{x}$ at which creditor $i$ is treated $f$-better than $j$, creditor $j$ is treated $f$-better than $k$ and creditor $k$ is treated $f$-better than $i$, it can be argued that the bilateral comparisons that arise from $f$ are meaningless. We feel that, when bilateral comparisons are meaningful, $f$-just allocations are more appealing than other allocations. This is so because the injustice of the latter is more evident in this case. It turns out that to ensure meaningful bilateral comparisons, the (monotone) bilateral principle $f$ has to have a consistent extension. The existence of such rule is equivalent to the existence of $f$-just allocations in all bankruptcy problems. The relation between the transitivity of the binary relation and consistency is stated formally as follows:

Theorem 3.2: Let $f$ be a monotone and anonymous bilateral principle and let $(E ; \mathbf{d})$ be a bankruptcy problem. $(E$; d) admits a unique $f$-just allocation if and only if for each allocation $\mathbf{x}$ in it, $\succ_{\mathbf{x}}$ is transitive.

In view of remark 2.1, we can interpret this theorem as saying that a monotone bilateral principle of justice can be extended to a consistent bankruptcy rule if and only if it provides meaningful bilateral comparisons. The proof appears in the following section.

Young (1987) considered bankruptcy rules that have a parametric representation: Let $g(d, \lambda)$ be a real valued function of two scalar variables $d$ and $\lambda$, where $d \geq 0$ and $\lambda$ ranges over some closed interval $[a, b]$ of the extended reals, and $g$ is continuous and non decreasing in $\lambda, g(d, a)=0$ and $g(d, b)=d$. A rule $\phi$ has a parametric representation, if there exists a function $g$ as above, which satisfies:

$$
\mathbf{x}=f(E ; \mathbf{d}) \quad \text { if and only if } \exists \lambda: \quad \forall i\left[x_{i}=g\left(d_{i}, \lambda\right), \sum_{i \in I} x_{i}=E\right] .
$$

Young (1987, Theorem 1) showed that the class of rules that have a parametric representation is identical to the class of symmetric, continuous, and consistent rules. This class is identical also to the class of anonymous, monotone, and consistent rules. Given a parametric representation $g$, of a rule $\phi$, a numerical presentation of $\succ_{\mathrm{x}}$ can be constructed. Define for each claim $d$, and for each amount $x \leq d$ the following values:

$$
\begin{aligned}
& \lambda_{*}(d, x)=\inf \{\lambda \mid g(d, \lambda)=x\} \\
& \lambda^{*}(d, x)=\sup \{\lambda \mid g(d, \lambda)=x\}
\end{aligned}
$$


Now, it is straightforward to verify that:

$$
i \succ_{\mathrm{x}} j \text { if and only if } \lambda_{*}\left(d_{i}, x_{i}\right)>\lambda^{*}\left(d_{j}, x_{j}\right) .
$$

This kind of interval representation of binary relations was analyzed by Fishburn (1970, pp. 18-22).

\section{On Average Consistency or Consistency on Average}

An appealing feature of $f$-just allocations is that no creditor can complain about being treated worse than any other creditor. However, as stated above, for some bankruptcy problems $f$-just allocations cannot be found. In this section, we present a weaker notion of consistency and $f$-justice that is compatible with any bilateral principle of justice.

Let $(E ; \mathbf{d})$ be an $n$-creditor bankruptcy problem and let $f$ be a monotone and anonymous bilateral principle. An allocation $\mathbf{x}^{*} \in A(E ; \mathbf{d})$ is said to be $f$-just on average (or average $f$-just) if for all creditors $i$.

$$
\sum_{j \neq i}\left[f_{i}\left(x_{i}^{*}+x_{j}^{*} ;\left(d_{i}, d_{j}\right)\right)-x_{i}^{*}\right]=0
$$

In order to understand this definition, note that $x_{i}^{*}$ is the amount awarded to creditor $i$ at allocation $\mathbf{x}^{*}$ and similarly for $x_{j}^{*} \cdot f_{i}\left(x_{i}^{*}+x_{j}^{*} ;\left(d_{i}, d_{j}\right)\right)$ is the amount $i$ should receive if $i$ and $j$ were to divide the amount $x_{i}^{*}+x_{j}^{*}$ they received $f$-equally between them. Hence $f_{i}\left(x_{i}^{*}+x_{j}^{*} ;\left(d_{i}, d_{j}\right)\right)-x_{i}^{*}$ is the amount that creditor $j$ should give creditor $i$ in order to justly divide the sum assigned to them. It can be interpreted as the amount $j$ owes $i$. $f$-justice on average requires from an allocation that the total debt of each creditor be zero. Clearly, this is a weaker condition than $f$-justice, which requires that no one should owe anything to anyone. The concept of average $f$-justice is inspired by Maschler and Owen's (1989) 2-consistency in hyperplane games.

The following result will allow us to provide a well-defined bankruptcy rule:

Theorem 4.1: Let $f$ be a monotone and anonymous bilateral principle. For every bankruptcy problem there exists a unique $f$-just on average allocation.

This proposition enables us to define the average $f$-just rule as the rule that assigns to each bankruptcy problem its unique average $f$-just allocation. The next proposition states that the average $f$-just rule inherits the basic properties of the bilateral principle $f$.

Proposition 4.2: Let $f$ be a monotone and anonymous bilateral principle. Then, the average $f$-just rule is monotone and anonymous. 


\section{Proof of Theorem 4.1:}

Lemma 4.3: If a rule is monotone then it is continuous in the estate.

Proof: Let $\left\{\left(E_{n} ; \mathbf{d}\right)\right\}_{n=1}^{\infty}$ be a sequence of bankruptcy problems that converges to $(E ; d)$ and let $\phi$ be a monotone rule. By monotonicity, for all $n \geq 1$ and for all $i \in I$, $0 \leq\left|\phi_{i}\left(E_{n} ; \mathbf{d}\right)-\phi_{i}(E ; \mathbf{d})\right| \leq\left|E_{n}-E\right|$. Since $\left\{E_{n}\right\}_{n=1}^{\infty}$ converges to $E$, it follows that $\left\{\phi_{i}\left(E_{n} ; \mathbf{d}\right)\right\}_{n=1}^{\infty}$ converges to $\phi_{i}(E ; \mathbf{d})$ for all $i \in I$ and therefore $\left\{\phi\left(E_{n} ; \mathbf{d}\right)\right\}_{n=1}^{\infty}$ converges to $\phi(E ; \mathbf{d})$.

Let $(E$; d) be an $n$-creditor bankruptcy problem and let $f$ be a monotone and anonymous bilateral principle. Define the following function:

$$
T: A(E ; \mathbf{d}) \rightarrow A(E ; \mathbf{d}) \mid T(\mathbf{x})=\mathbf{t}
$$

where

$$
t_{i}=(n-1)^{-1} \sum_{j \neq i} f_{i}\left(x_{i}+x_{j} ;\left(d_{i}, d_{j}\right)\right)
$$

and $n$ is the number of creditors in $(E ; \mathbf{d})$.

In order to see that $T$ maps allocations of $(E ; \mathbf{d})$ into allocations, note that for all $i$ and $j$ in $I$ and for all $\mathbf{x} \in A(E ; \mathbf{d}), 0 \leq f_{i}\left(x_{i}+x_{j} ;\left(d_{i}, d_{j}\right)\right) \leq d_{i}$. Hence, $0 \leq(n-1)^{-1} \sum_{j \neq i} f_{i}\left(x_{i}+x_{j} ;\left(d_{i}, d_{j}\right)\right) \leq d_{i}$. Moreover, $\sum_{i \in I}(n-1)^{-1} \sum_{j \neq i} f_{i}\left(x_{i}+x_{j}\right.$; $\left.\left(d_{i}, d_{j}\right)\right)=(n-1)^{-1} \sum_{i \in I} \sum_{i<j}\left[f_{i}\left(x_{i}+x_{j} ;\left(d_{i}, d_{j}\right)\right)+f_{j}\left(x_{i}+x_{j} ;\left(d_{i}, d_{j}\right)\right)\right]=$ $(n-1)^{-1} \sum_{i \in I} \sum_{i<j}\left(x_{i}+x_{j}\right)=\sum_{i \in I} x_{i}=E$.

Clearly, the set of allocations $A(E ; \mathbf{d})$ is compact and convex. Moreover, since $f$ is monotone, Lemma 4.3 implies that $T$ is continuous. Hence by Brouwer's fixed-point theorem, $T$ has a fixed point. Clearly, any fixed point of $T$ is an average $f$-just allocation of $(E ; \mathbf{d})$ and conversely, any allocation in $(E ; \mathbf{d})$ that is $f$-just on average, is a fixed point of $T$.

For any $\mathbf{x}$ in $\mathbb{R}^{I}$ let $\|\mathbf{x}\|=\sum_{i \in I}\left|x_{i}\right|$. The uniqueness part will follow from the following lemma:

Lemma 4.4: Let $\mathbf{x}$ and $\mathbf{y}$ be two allocations in $(E ; \mathbf{d})$. If $\mathbf{x} \neq \mathbf{y}$ then $\|T(\mathbf{x})-T(\mathbf{y})\|<\|\mathbf{x}-\mathbf{y}\|$.

Proof: Let $\mathbf{x}$ and $\mathbf{y}$ be two distinct allocations in $(E ; \mathbf{d})$. By definition of $T$ and by the triangle inequality we have:

$$
\begin{aligned}
\left|T_{i}(\mathbf{x})-T_{i}(\mathbf{y})\right| & =\left|(n-1)^{-1} \sum_{j \neq i} f_{i}\left(x_{i}+x_{j} ;\left(d_{i}, d_{j}\right)\right)-(n-1)^{-1} \sum_{j \neq i} f_{i}\left(y_{i}+y_{j} ;\left(d_{i}, d_{j}\right)\right)\right| \\
& \leq(n-1)^{-1} \sum_{j \neq i}\left|f_{i}\left(x_{i}+x_{j} ;\left(d_{i}, d_{j}\right)\right)-f_{i}\left(y_{i}+y_{j} ;\left(d_{i}, d_{j}\right)\right)\right| .
\end{aligned}
$$


Summing over all creditors $i$ we have:

$$
\begin{aligned}
\|T(\mathbf{x})-T(\mathbf{y})\| \leq & (n-1)^{-1} \sum_{i \in I} \sum_{j \neq i}\left|f_{i}\left(x_{i}+x_{j} ;\left(d_{i}, d_{j}\right)\right)-f_{i}\left(y_{i}+y_{j} ;\left(d_{i}, d_{j}\right)\right)\right| \\
\leq & (n-1)^{-1} \sum_{i \in I} \sum_{j<i}\left[\left|f_{i}\left(x_{i}+x_{j} ;\left(d_{i}, d_{j}\right)\right)-f_{i}\left(y_{i}+y_{j} ;\left(d_{i}, d_{j}\right)\right)\right|\right. \\
& \left.+\left|f_{j}\left(x_{i}+x_{j} ;\left(d_{i}, d_{j}\right)\right)-f_{j}\left(y_{i}+y_{j} ;\left(d_{i}, d_{j}\right)\right)\right|\right] .
\end{aligned}
$$

By monotonicity, the terms inside the absolute values have the same sign, hence

$$
\begin{aligned}
\leq & (n-1)^{-1} \sum_{i \in I} \sum_{j<i}\left[\mid f_{i}\left(x_{i}+x_{j} ;\left(d_{i}, d_{j}\right)\right)-f_{i}\left(y_{i}+y_{j} ;\left(d_{i}, d_{j}\right)\right)+f_{j}\left(x_{i}+x_{j} ;\left(d_{i}, d_{j}\right)\right)\right. \\
& \left.\quad-f_{j}\left(y_{i}+y_{j} ;\left(d_{i}, d_{j}\right)\right) \mid\right],
\end{aligned}
$$

and since $f$ assigns allocations,

$$
\leq(n-1)^{-1} \sum_{i \in I} \sum_{j<i}\left|\left(x_{i}+x_{j}\right)-\left(y_{i}+y_{j}\right)\right| .
$$

By the triangle inequality, $\left|\left(x_{i}+x_{j}\right)-\left(y_{i}+y_{j}\right)\right| \leq\left|x_{i}-y_{i}\right|+\left|x_{j}-y_{j}\right|$ and since $\mathbf{x} \neq \mathbf{y}$, for some pair of creditors $i$ and $j$ this last inequality is strict. Therefore,

$$
\begin{aligned}
\|T(\mathbf{x})-T(\mathbf{y})\| & <(n-1)^{-1} \sum_{i \in I} \sum_{j<i}\left[\left|x_{i}-y_{i}\right|+\left|x_{j}-y_{j}\right|\right] \\
& =1 / 2(n-1)^{-1} \sum_{i \in I} \sum_{j \neq i}\left[\left|x_{i}-y_{i}\right|+\left|x_{j}-y_{j}\right|\right] \\
& =1 / 2(n-1)^{-1}\left[\sum_{i \in I}(n-1)\left|x_{i}-y_{i}\right|+\sum_{i \in I} \sum_{j \neq i}\left|x_{j}-y_{j}\right|\right] \\
& =1 / 2(n-1)^{-1}\left[\sum_{i \in I}(n-2)\left|x_{i}-y_{i}\right|+\sum_{i \in I} \sum_{j \in J}\left|x_{j}-y_{j}\right|\right] \\
& =1 / 2(n-1)^{-1}[(n-2)\|\mathbf{x}-\mathbf{y}\|+n\|\mathbf{x}-\mathbf{y}\|] \\
& =\|\mathbf{x}-\mathbf{y}\| .
\end{aligned}
$$

It follows immediately from Lemma 4.4 that if $\mathbf{x}$ and $\mathbf{y}$ are fixed points of $T$, they must be equal. This completes the proof of Theorem 4.1 .

Proof of Proposition 4.2: Since the anonymity part is trivial, we only prove the monotonicity of the average $f$-just rule. Let $(E$; d $)$ be a bankruptcy problem and let $0 \leq E^{\prime}<E$. Denote by $\mathbf{x}$ and by $\mathbf{y}$ the average $f$-just allocations of $(E ; \mathbf{d})$ and $\left(E^{\prime} ; \mathbf{d}\right)$ respectively. Assume by contradiction that $y_{k}>x_{k}$ for some creditor $k$. By an argument analogous to the one in Lemma 4.4, we have

$$
\|T(\mathbf{x})-T(\mathbf{y})\| \leq(n-1)^{-1} \sum_{i \in I} \sum_{j<i}\left|\left(x_{i}+x_{j}\right)-\left(y_{i}+y_{j}\right)\right| .
$$


By the triangle inequality, $\left|\left(x_{i}+x_{j}\right)-\left(y_{i}+y_{j}\right)\right| \leq\left|x_{i}-y_{i}\right|+\left|x_{j}-y_{j}\right|$ for each pair of creditors $i$ and $j$. But since for some creditor $k y_{k}>x_{k}$, and since $\sum_{i \in I} x_{i}>\sum_{i \in I} y_{i}$, for some pair of creditors $i$ and $j$ the triangle inequality is strict. Therefore,

$$
\|T(\mathbf{x})-T(\mathbf{y})\|<(n-1)^{-1} \sum_{i \in I} \sum_{j<i}\left[\left|x_{i}-y_{i}\right|+\left|x_{j}-y_{j}\right|\right] .
$$

Again, by the same argument as in Lemma 4.4, we get

$$
\|T(\mathbf{x})-T(\mathbf{y})\|<\|\mathbf{x}-\mathbf{y}\|
$$

contradicting the fact that $\mathbf{x}$ and $\mathbf{y}$ are both fixed points.

Since $f$-just allocations are also $f$-just on average, Theorem 4.1 yields the well-known result that if $f$-just allocations exist, they are unique. The next proposition shows that we can use the operator $T$, defined in the proof of Theorem 4.1, to define a dynamic process that always converges to the average $f$-just allocation.

Proposition 4.5: Let $\mathbf{x}_{0}$ be an allocation in $(E ; \mathbf{d})$ and define inductively $\mathbf{x}_{t}=T\left(x_{t-1}\right)$ for $t>0 .\left\{\mathbf{x}_{t}\right\}$ converges to the average $f$-just allocation.

Proof: ${ }^{7}$ Since $\mathbf{x}_{t} \in A(E ; \mathbf{d})$ and $A(E ; \mathbf{d})$ is bounded, $\left\{\mathbf{x}_{t}\right\}$ has a convergent subsequence $\left\{\mathbf{x}_{t(k)}\right\}$. Let $\mathbf{y}$ be the limit of this subsequence. Since $A(E ; \mathbf{d})$ is closed, $\mathbf{y} \in A(E ; \mathbf{d})$. It is sufficient to show that $\mathbf{y}$ is the unique $f$-just on average allocation in $(E ; \mathbf{d})$, which will be denoted by $\mathbf{x}$. It follows from Lemma 4.4 that $\left\{\left\|\mathbf{x}_{t}-\mathbf{x}\right\|\right\}$ is a non-increasing sequence of non-negative real numbers, hence it must have a limit, i.e., $\left\|\mathbf{x}_{t}-\mathbf{x}\right\| \rightarrow a$. Since $\left\{\mathbf{x}_{t(k)}\right\}$; and $\left\{T\left(\mathbf{x}_{t(k)}\right\}\right.$ are subsequences of $\left\{\mathbf{x}_{t}\right\}$ we must have $\left\|\mathbf{x}_{t(k)}-\mathbf{x}\right\| \rightarrow\|\mathbf{y}-\mathbf{x}\|=a$ and by continuity of $T,\left\|T\left(\mathbf{x}_{t(k)}\right)-\mathbf{x}\right\| \rightarrow$ $\|T(\mathbf{y})-\mathbf{x}\|=a$. But then $\|\mathbf{y}-\mathbf{x}\|=\|T(\mathbf{y})-\mathbf{x}\|=\|T(\mathbf{y})-T(\mathbf{x})\|$ and Lemma 4.4 implies that $\mathbf{y}=\mathbf{x}=T(\mathbf{x})$.

We can now proceed to the proof of Theorem 3.2.

Proof of Theorem 3.2: The proof follows from the following lemmas:

Lemma $4.6:^{8}$ Let $f$ be a monotone and anonymous bilateral principle. Let $(E ; \mathbf{d})$ be a bankruptcy problem with at least three creditors and $\mathbf{x}$ an allocation. If there exists an $f$-just allocation in each 3-creditor reduced problem with respect to $\mathbf{x}$ then $>_{\mathrm{x}}$ is transitive.

\footnotetext{
7 This proof is due to Sjaak Hurkens.

8 Lemma 4.6 is similar to Dagan, Serrano, and Volij (1994, Lemma 3.4), but is slightly stronger.
} 
Proof: Let $(E ; \mathbf{d})$ be a bankruptcy problem and let $\mathbf{x}$ be an allocation. Let $i, j$ and $k$ be three creditors such that $i \succ_{\mathrm{x}} j$ and $j \succ_{\mathrm{x}} k$. Let $\left(E^{\prime} ; \mathbf{d}^{\prime}\right)$ be the reduced bankruptcy problem of $\{i, j, k\}$ with respect to $\mathbf{x}$, i.e., $\left(E^{\prime} ; \mathbf{d}^{\prime}\right):=\left(x_{i}+x_{j}+x_{k}\right.$; $\left.\left(d_{i}, d_{j}, d_{k}\right)\right)$. Let $\mathbf{x}^{*}$ be the $f$-just allocation of this problem. It must be the case that $x_{k}<x_{k}^{*}$. Otherwise, since $j \succ_{\mathrm{x}} k$, Lemma 3.1 implies $x_{j}>x_{j}^{*}$ and since $j \succ_{\mathrm{x}} j$, the same lemma implies $x_{i}>x_{i}^{*}$ contradicting the fact that $x_{i}+x_{j}+x_{k}=x_{i}^{*}+$ $x_{j}^{*}+x_{k}^{*}$. Hence, $x_{k}<x_{k}^{*}$. Analogously, it must be that $x_{i}>x_{i}^{*}$. Hence by Lemma $3.1 i \succ_{\mathrm{x}} k$. This completes the proof of Lemma 4.6.

Lemma 4.7: Let $f$ be a monotone and anonymous bilateral principle. Let $(E$; $\mathbf{d})$ be an $n$-creditor bankruptcy problem and let $\mathbf{x}$ be the average $f$-just allocation. If $\succ_{\mathrm{x}}$ is transitive, then $\mathbf{x}$ is an $f$-just allocation.

Proof: Assume by contradiction that $\mathbf{x}$ is not $f$-just and $\succ_{\mathbf{x}}$ is transitive. Since $\mathbf{x}$ is not $f$-just, there are two creditors $i$ and $j$ such that $i \succ_{\mathbf{x}} j$ and since $\mathbf{x}$ is $f$-just on average, there exists a creditor $k$ such that $j \succ_{\mathbf{x}} k$. By transitivity $i \succ_{\mathbf{x}} k$ and by irreflexivity, $i \neq k$. Again, since $\mathbf{x}$ is $f$-just on average there exists a creditor $m$ such that $k \succ_{\mathrm{x}} m$. The above arguments show that $m$ is different from the previous creditors. Applying these arguments $n$ times will contradict the fact that there are $n$ creditors. This completes the proof of Lemma 4.7.

To see that Theorem 3.2 follows from Lemma 4.6 and 4.7 , let $(E$; d) be a bankruptcy problem. If for each allocation $\mathbf{x} \succ_{\mathrm{x}}$ is transitive, then it is transitive when $\mathbf{x}$ is an $f$-just on average allocation, which by Theorem 4.1 exists and is unique. But in this case Lemma 4.7 ensures that $\mathbf{x}$ is the $f$-just allocation of $(E ; \mathbf{d})$. Conversely, if $\mathbf{x}$ is an $f$-just allocation in $(E ; \mathbf{d})$, then $\mathbf{x} \mid J$ is an $f$-just allocation in the reduced bankruptcy problem of $J$ with respect to $\mathbf{x}$ for all $J$ containing exactly 3 creditors. Therefore, by Lemma $4.6 \succ_{\mathbf{x}}$ is transitive for all allocations $\mathbf{x}$.

Corollary 4.8: Let $f$ be a monotone and anonymous bilateral principle. For each 3 -creditor bankrupcty problem there exists an $f$-just allocation if and only if for each bankruptcy problem there exists an $f$-just allocation.

Proof: Lemma 4.6 says that if for each 3-creditor bankruptcy problems there exists an $f$-just allocation then $\succ_{\mathrm{x}}$ is transitive for all allocations $\mathbf{x}$ and Theorem 3.2 ensures that in this case there exists an $f$-just allocation in each bankruptcy problem. The other direction is immediate.

This corollary says that the answer to the question of the existence of a consistent extension to a specific bilateral principle lies in the family of 3 -creditor problems. If there is no consistent extension of a bilateral principle $f$, then we must be able to find a 3-creditor problem with no $f$-just allocation.

Theorem 3.2 states that there is a connection between the existence of $f$-just allocations and the transitivity of the relations $\succ_{\mathrm{x}}$. On the other hand, a rule may well be consistent while the weak relations $\succeq_{\mathrm{x}}$ are not transitive. To see this, consider the following bankruptcy problem: $(E ; \mathbf{d}):=(400 ;(300,200,100))$ and the 
following allocation: $\mathbf{x}=(160,140,100)$. When $f$ is the constrained equal award rule it is easy to see that $2 \geq_{x} 3,3 \geq_{x} 1$ but $1 \succ_{x} 2$. However, the following theorem states that there is some relation between the transitivity of the weak relations $\succeq_{\mathrm{x}}$ and the strict monotonicity of the bilateral principle $f$.

Theorem 4.9: Let $f$ be a monotone and anonymous bilateral principle. $f$ is strictly monotone and for each bankruptcy problem there exists an $f$-just allocation if and only if for each bankruptcy problem with no zero creditors and for all allocations $\mathbf{x}$ in it $\succeq_{\mathbf{x}}$ is transitive.

Proof: "Only if": Let $(E ; \mathbf{d})$ be a bankruptcy problem with no zero creditors, let $\mathbf{x}$ be an allocation in it and let $f$ be a strictly monotone bilateral principle. We shall show that $\succeq_{\mathrm{x}}$ is transitive whenever there is an $f$-just allocation in $(E$; d). Assume that there are three creditors $i, j$ and $k$ such that $i \succeq_{\mathrm{x}} j$ and $j \succeq_{\mathrm{x}} k$. Consider the reduced bankruptcy problem of $i, j$ and $k$ with respect to $\mathbf{x}$ and denote by $\mathbf{x}^{*}$ its $f$-just allocation (by assumption, this allocation exists). Since $i \succeq_{\mathrm{x}} j$, Lemma 3.1 implies that either $x_{i} \geq x_{i}^{*}$ or $x_{j} \leq x_{j}^{*}$. Since $j \geq_{\mathrm{x}} k$, Lemma 3.1 implies that either $x_{j} \geq x_{j}^{*}$ or $x_{k} \leq x_{k}^{*}$. Assume by contradiction that $k>_{\mathrm{x}} i$. Then, again by Lemma 3.1 eithet $x_{i}<x_{i}^{*}$ or $x_{k}>x_{k}^{*}$. But it follows from the previous inequalities that in either case $x_{j}=x_{j}^{*}$. So if $x_{i}<x_{i}^{*}$, then $x_{i}+x_{j}<x_{i}^{*}+x_{j}^{*}$ and by strict monotonicity of $f$ we have $f_{j}\left(x_{i}+x_{j} ;\left(d_{i}, d_{j}\right)\right)<f_{j}\left(x_{i}^{*}+x_{j}^{*} ;\left(d_{i}, d_{j}\right)\right)=$ $x_{j}^{*}=x_{j}$, contradicting the fact that $i \geq_{\mathrm{x}} j$. Similarly, if $x_{k}>x_{k}^{*}$ then $x_{j}+x_{k}>x_{j}^{*}+x_{k}^{*}$. Hence by strict monotonicity of $f$ we have $f_{j}\left(x_{j}+x_{k} ;\left(d_{j}, d_{k}\right)\right)>$ $f_{j}\left(x_{j}^{*}+x_{k}^{*} ;\left(d_{j} ; d_{k}\right)\right)=x_{j}^{*}=x_{j}$, contradicting the assumption that $j \succeq_{\mathbf{x}} k$.

"If": Assume $\geq_{\mathbf{x}}$ is transitive for every bankruptcy problem $(E ; \mathbf{d})$ with no zero creditors and allocation $\mathbf{x}$ in it, and that $f$ is not strictly monotone. Then there are two 2-creditor bankruptcy problems $(E ; \mathbf{d})$ and $\left(E^{\prime} ; \mathbf{d}\right)$ with $0 \leq E^{\prime}<E$, $I=\{i, j\}, \mathbf{d}>\mathbf{0}$ with $f_{i}(E ; \mathbf{d})=f_{i}\left(E^{\prime} ; \mathbf{d}\right)=x_{i}$ and $x_{j}=f_{j}(E ; \mathbf{d})>f_{j}\left(E^{\prime} ; \mathbf{d}\right)=y_{j}$. Let $\left(E^{*} ; \mathbf{d}^{*}\right)=\left(x_{i}+x_{j}+y_{j} ;\left(d_{i}, d_{j}, d_{j}\right)\right)$ and let $\mathbf{x} \in A\left(E^{*} ; \mathbf{d}^{*}\right)$ be the allocation $\left(x_{i}, x_{j}, y_{j}\right)$. By construction $2 \succeq_{\mathrm{x}} 1,1 \succeq_{\mathrm{x}} 3$ and by anonymity $3 \succ_{\mathrm{x}} 2$, which contradicts the transitivity of $\succeq_{\mathbf{x}}$. Finally, we need to show that for each bankruptcy problem, there exists an $f$-just allocation. By corollary 4.8 , it is enough to show this for each 3 -creditor bankruptcy problem. So let $(E ; \mathbf{d})$ be a 3 -creditor bankruptcy problem (call them $i, j$, and $k$ ) and let $\mathbf{x}$ be an allocation in it. Assume that $i \succ_{\mathrm{x}} j$ and $j \succ_{\mathrm{x}} k$. Since in all allocations any two creditors, one of which is a zero, are treated $f$-equally, it follows that there is no zero creditor in $(E ; \mathbf{d})$. By assumption, then, $\succeq_{\mathrm{x}}$ is transitive which in turn implies that $i \succ_{\mathrm{x}} k$. Hence, $\succ_{\mathrm{x}}$ is transitive. Since $\mathbf{x}$ was chosen to be an arbitrary allocation in $(E ; \mathbf{d}), \succ_{\mathbf{x}}$ is transitive for all allocations $\mathbf{x}$ in it and by Theorem 3.2, there is an $f$-just allocation in $(E ; \mathbf{d})$.

\section{Discussion}

In this section we discuss the role of the two basic properties of bilateral principles assumed in our results, namely anonymity and monotonicity. 
The anonymity of the bilateral principle is actually not needed for any of the above results, with the only exception of the "if" part of Theorem 4.9. We still assumed anonymity since it is a natural assumption in the context of justice and because it simplifies notation.

The role of monotonicity is more critical and it cannot be relaxed if we want a bilateral principle to univocally define an average consistent rule. Moreover, for most of our results, it cannot be replaced by the continuity in the estate. Take for example Theorem 4.1. It is clear from its proof that if $f$ is an anonymous and continuous bilateral principle, then every bankruptcy problem admits an $f$-just on average allocation. These assumptions, however, do not guarantee the uniqueness of such an allocation. Moreover, only the "if" part of Theorem 3.2 remains valid if we replace monotonicity by continuity. All this means that, if the bilateral principle is not monotonic, there might be several distinct rules that assign to each bankruptcy problem an average $f$-just allocation, and more importantly there might be several $f$-just allocations for some bankruptcy problems. Hence, even with continuity we cannot preclude the possibility of there being more than one consistent extension of a bilateral principle.

On the other hand, Young (1987) showed that if $\phi$ is an anonymous, continuous and consistent rule then $\phi$ (and a fortiori its associated bilateral principle) is monotone. This means that there is no chance of finding a continuous, anonymous and consistent extension of a bilateral principle which is not monotonic. That is, among the various consistent extensions of a non-monotonic bilateral principle (if there is one), there is not even one which is anonymous and continuous. One remark is in order, Young (1987) assumes that the set of potential creditors is countably infinite, i.e., he assumes "variable number of agents". Our results do not need this assumption. If we restrict ourselves to a finite set of potential creditors, then Young's result is no longer valid and there might be one or more continuous and anonymous consistent extensions of a given non-monotonic bilateral principle.

We have already mentioned that the bilateral principle associated with the MP rule cannot be extended in a consistent way. Its average consistent extension, however, is well-defined by virtue of Theorem 4.1. Two questions come to mind: How does the MP-just on average rule relate to the MP-rule? Is there any concise expression of the former? We were not able to find any simple formula for the MP-just on average rule. We can only say that, in view of Young (1987 Theorem 1), this rule has no parametric representation. With respect to the first question, the MP-rule and the MP-just on average rule are different. Although they

Table 5.1. MP and MP-just on average allocations for $(E ; \mathbf{d})=(E ; 100,200300)$

\begin{tabular}{llll}
\hline allocation $E$ & 150 & 300 & 450 \\
\hline MP-allocation & $(37.5 ; 56.25 ; 56.25)$ & $(50 ; 100 ; 150)$ & $(75 ; 150 ; 225)$ \\
MP-just on average & $(50 ; 50 ; 50)$ & $(64.5 ; 109.2 ; 126.2)$ & $(75 ; 150 ; 225)$ \\
MP-just allocation & $(50 ; 50 ; 50)$ & does not exist & $(75 ; 150 ; 225)$ \\
\hline
\end{tabular}


coincide for some bankruptcy problems, they differ significantly for some others. Table 5.1 gives the MP and the MP-just on average allocations of three different problems.

\section{Conclusion}

The motivation for this paper is to find conditions on bilateral principles that assure the possibility of extending them to a consistent $n$-creditor rule. However, an alternative motivation can be found. Since a characterization of the bilateral principles mentioned above is equivalent to a characterization of the consistent rules induced by them, our main result can be viewed as an equivalence theorem for the class of monotone and consistent rules. This equivalence theorem, in contrast to Young's, characterizes the monotone and consistent rules by properties that relate to allocations that the rules do not recommend. Although different in nature, our results provide a new interpretation of parametric representations of bankruptcy rules.

Many bilateral principles do not have a consistent extension (for example the 2-creditor MP rule). The introduction of consistency on average sees a natural alternative. We believe that further study of these rules may be of interest.

\section{References}

Aumann RJ, Maschler M(1985) Game theoretic analysis of a bankruptcy problem from the talmud. Journal of Economic Theory 36: 195-213

Dagan N, Volij O(1993) The bankruptcy problem, a cooperative bargaining approach. Mathematical Social Sciences 26: 287-297

Dagan N, Serrano R, Volij O (1994) A non-cooperative view of consistent bankruptcy rules. Discussion Paper 9411, CentER for Economic Research Tilburg, The Netherlands

Fishburn PC (1970) Utility theory for decision making, John Wiley and Sons, NY

Lensberg T (1987) Stability and collective rationality. Econometrica 55: 935-961

Maschler M Owen G (1989) The consistent shapley value for hyperplane games. International Journal of Game Theory 18: 389-407

Peleg B (1986) On the reduced game property and its converse. International Journal of Game Theory 15: $187-200$

Sen A (1969) Quasi-transitivity, rational choice and collective decisions. Review of Economic Studies 36: 381-393

Thomson W (1990) The consistency principle. In: Ichiishi T, Neyman A, Tauman Y (Eds) Game Theory and Applications. Academic Press San Diego CA

Thomson W (1994) Consistent extensions. Mathematical Social Sciences 28: 35-49

Young HP (1987) On dividing an amount according to individual claims or liabilities. Math Opr Res 12: $398-414$

Young HP (1988) Distributive justice in taxation. Journal of Economic Theory 44: 321-335

Received March 1994

Revised version August 1994

Final version January 1995 